\title{
Irreversible electroporation of hepatocellular carcinoma: patient selection and perspectives
}

\author{
This article was published in the following Dove Press journal: \\ Journal of Hepatocellular Carcinoma \\ 13 March 2017 \\ Number of times this article has been viewed
}

\begin{abstract}
Asha Zimmerman'
David Grand ${ }^{2}$

Kevin P Charpentier ${ }^{1}$

'Department of Surgery, ${ }^{2}$ Department of Radiology, Rhode Island Hospital, Brown University, Providence, RI, USA
\end{abstract}

Correspondence: Kevin P Charpentier Department of Surgery, Rhode Island Hospital, Brown University, 2 Dudley Street, Suite 370, Providence, RI 02905, USA

Tel + I 40I 2280560

Fax +I 40I 2280636

Email kcharpentier@usasurg.org
Abstract: Irreversible electroporation (IRE) is a novel form of tissue ablation that uses high-current electrical pulses to induce pore formation of the cell lipid bilayer, leading to cell death. The safety of IRE for ablation of hepatocellular carcinoma (HCC) has been established. Outcome data for ablation of HCC by IRE are limited, but early results are encouraging and suggest equivalency to the outcomes obtained for thermal ablation for appropriately selected, small $(<3 \mathrm{~cm})$ tumors. Long-term oncologic efficacy and histopathologic response data have not been published, and therefore, application of IRE for the treatment of HCC should still be viewed with caution.

Keywords: ablation, liver, tumors, IRE, hepatic

\section{Background}

Liver cancer is the second most common cause of cancer-related death worldwide, causing an estimated 746,000 deaths in 2012 . It is responsible for $9 \%$ of the global cancer mortality burden and carries a high mortality to incidence ratio of 0.95 . Hepatocellular carcinoma (HCC) comprises $80 \%$ of the primary hepatic cancers. ${ }^{1}$ Thermal ablation using either radiofrequency ablation (RFA) or microwave ablation (MWA) has been established as a standard of care therapy for selected, small hepatocellular cancers that are not amenable to surgical resection. ${ }^{2,3}$ Thermal ablation is limited by both heat sink and collateral damage., ${ }^{4,5}$ Irreversible electroporation (IRE) is a novel, nonthermal form of tumor ablation that is not affected by heat sink and may result in less collateral damage based on its mechanism of action. ${ }^{6,7}$ IRE relies on short pulses of high-frequency energy to induce pores in the lipid bilayer of cells, ${ }^{8}$ leading to cell death via apoptosis. Acellular elements within the treatment field are spared, resulting in preserved parenchymal architecture. ${ }^{9}$

Selecting patients suitable for IRE varies between institutions; however, common criteria can be found throughout the literature. Generally, principles of selection include patients who would otherwise be amenable to have local therapy with RFA or MWA but have tumors adjacent to structures that would cause either heat sink or collateral damage. Heat sink is the inability to achieve an adequate temperature for cell death $\left(60^{\circ} \mathrm{C}\right)$ due to close proximity of a large vessel. ${ }^{10,11}$ Conversely, due to the temperatures achieved with RFA, the opposite effect may cause collateral damage to normal surrounding structures, leading to injury to structures such as bile ducts or gallbladder., ${ }^{4,5}$

The only commercially available system for IRE is NanoKnife ${ }^{\circledR}$ (AngioDynamics, Queensbury, NY, USA). NanoKnife electrodes are housed in a 19-gauge 
probe. The system supports the use of 6 monopolar electrodes simultaneously that can be positioned either percutaneously or surgically to bracket the target tissue. After electrode positioning under either ultrasound or computed tomography (CT) guidance, delivery of electrical energy is calculated based on proprietary algorithms within the NanoKnife system. Treatment delivery must be accompanied by general anesthesia, paralysis, and cardiac synchronization in order to avoid muscle contractions and arrhythmias, respectively. ${ }^{12}$

In order to evaluate the safety and efficacy of IRE in the setting of HCC, a comprehensive literature search was done in PubMed by using the following keywords: "electroporation", "irreversible electroporation", and "irreversible electroporation hepatocellular carcinoma". A total of 3,567 articles were evaluated (Figure 1). Ninety six articles written in the English language referenced electroporation of $\mathrm{HCC}$ and were reviewed in detail. All the data regarding the safety and efficacy of IRE were extracted from the articles reviewed, and any relevant articles on the topics that were not captured through search but encountered through the literature review processes were also included.

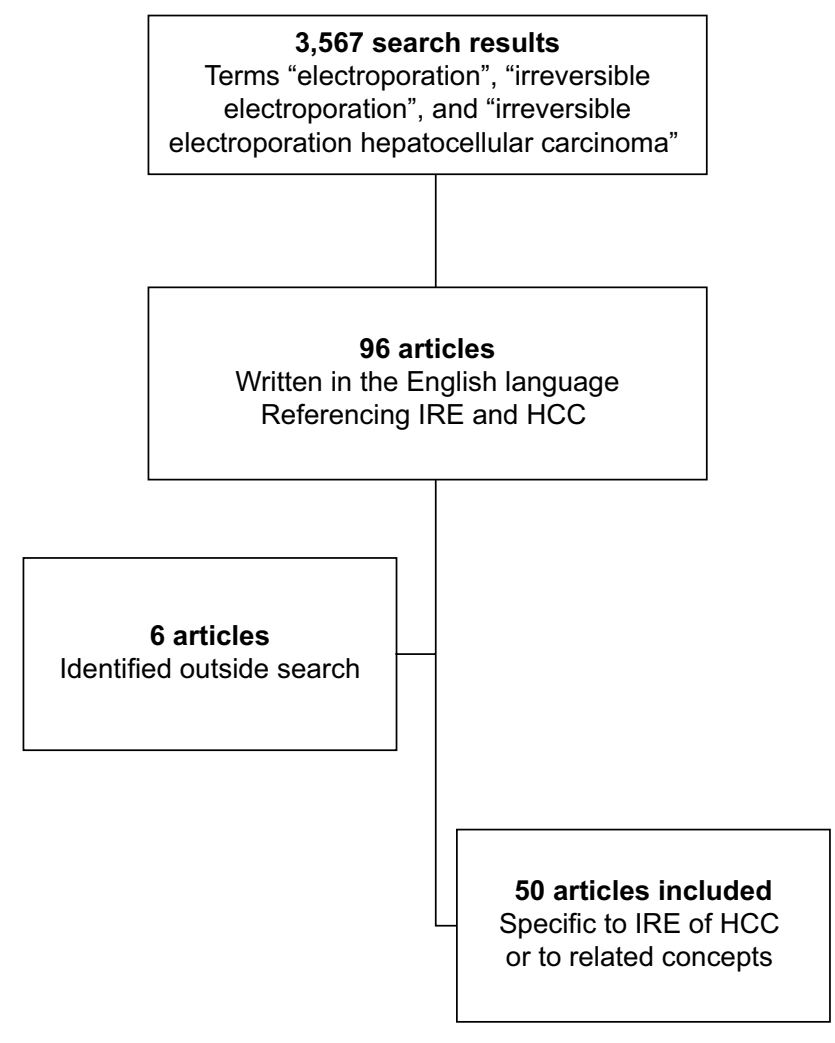

Figure I Flowchart of review process.

Abbreviations: HCC, hepatocellular carcinoma; IRE, irreversible electroporation.

\section{IRE for HCC}

\section{Safety}

In the early experience, IRE was hampered by cardiac toxicity and arrhythmias. ${ }^{12}$ Since the introduction of cardiac synchronization with IRE treatment delivery, ${ }^{13}$ the safety of IRE for liver ablation has been established in several case series and a systematic review. ${ }^{14-17}$

An assessment of vascular changes in close proximity to hepatic lesions treated with IRE was done by Dollinger et al. ${ }^{14}$ The authors noted that, in a total of 84 hepatic lesions (31 primary tumors and 53 secondary hepatic tumors), 191 venous structures were located within $1 \mathrm{~cm}$ of the ablation zone; $29 \%$ of these were encased by the ablation zone, $41 \%$ were abutting, and $30 \%$ were located within $1 \mathrm{~cm}$ of the zone of ablation. At follow-up, they noted that 19 (9.9\%) vessels had vascular changes such as partial $(n=2)$ and complete $(n=3)$ portal vein thrombosis and vessel narrowing $(n=14)$. At a further follow-up, they noted that thrombosis had resolved in 2 of 5 cases and that vessel narrowing had completely $(n=8)$ or partially resolved $(n=1)$ in 9 of 14 cases, concluding that venous structures in close proximity to ablation zones are minimally affected by IRE.

Dollinger et al also investigated the rate of bile duct injury following IRE in another study. ${ }^{15}$ They report on 55 bile ducts within $1 \mathrm{~cm}$ of the zone of ablation in 53 hepatic tumors; of these, 12 were HCC lesions. Proximity was defined as encased $(n=33)$, abutting $(n=14)$, and located within $1 \mathrm{~cm}$ of the zone of ablation $(n=8)$. On follow-up magnetic resonance imaging (MRI), they noted that 40 bile ducts remained unchanged, $8(14.5 \%)$ had bile duct narrowing, and a further $7(12.7 \%)$ had dilation. They noted that injuries were statistically more common in patients aged $>65$ years.

Dollinger et $\mathrm{al}^{16}$ also published a retrospective review of all of their adverse events after IRE of malignant liver tumors. Their cohort was comprised of 85 ablations in 114 tumors (24 patients with $45 \mathrm{HCC}$ lesions) and had a complication rate of $26 \%$ ( 22 of 85 ). They noted that their most common major complication was postablative abscess (4.7\%), which was noted to affect patients with a bilioenteric anastomoses more frequently than those without (Table 1). They also published results with IRE of 65 hepatic tumors, of which 33 were HCC. In this series, the authors reported an overall complication rate of $27.5 \% .{ }^{18}$ Complications included the following: partial portal vein thrombosis in a cirrhotic patient requiring anticoagulation $(n=1)$, intraperitoneal bleed requiring intensive care unit admission and transfusion $(n=1)$, abscess formation $(n=4)$, hematomas $(n=6)$, and small, clinically insignificant 
Table I Complications following IRE of liver tumors

\begin{tabular}{|c|c|c|c|c|c|c|}
\hline Reference & $\begin{array}{l}\text { Number } \\
\text { of patients }\end{array}$ & $\begin{array}{l}\text { Number } \\
\text { of tumors }\end{array}$ & Histology & Approach & $\begin{array}{l}\text { Complication } \\
\text { rate }\end{array}$ & Complications \\
\hline Niessen et al ${ }^{18}$ & 34 & 65 & $33,{ }^{a} 5,{ }^{b} 27^{c}$ & Percutaneous & $27.5 \%(14 / 51)^{d}$ & $\begin{array}{l}\text { PVT }(n=I) \text {; bleeding }(n=1) \text {, abscess }(n=4) \text {, } \\
\text { hematoma }(n=6) \text {, PTX }(n=2)\end{array}$ \\
\hline Cannon et al ${ }^{19}$ & 44 & 48 & $14,{ }^{\mathrm{a}} 30^{\mathrm{c}}$ & Mixed & $10 \%(5 / 48)^{d}$ & $\begin{array}{l}\text { Leukocytosis }(n=I) \text {, UTI }(n=I) \text {, dehydration } \\
(n=I) \text {, biliary stent occlusion }(n=I) \text {, cholangitis } \\
(n=I) \text {, renal failure }(n=I) \text {, abdominal pain }(n=I) \text {, } \\
\text { flank pain }(n=I) \text {, neurogenic bladder }(n=I)\end{array}$ \\
\hline Cheung et $\mathrm{a}^{20}$ & 11 & 18 & $18^{a}$ & Percutaneous & $0 \%(0 / I I)^{d}$ & $7 / I I$ pain, $4 / 11$ urinary retention \\
\hline Bhutiani et $\mathrm{al}^{21}$ & 30 & $\mathrm{~N} / \mathrm{A}$ & $30^{\mathrm{a}}$ & Surgical & $27 \%(8 / 30)^{d}$ & $\begin{array}{l}\text { Pleural effusion I7\% }(n=5) \text {, PVT } 3 \%(n=1) \text {, ascites } \\
17 \%(n=5) \text {, }\end{array}$ \\
\hline Eller et $\mathrm{al}^{22}$ & 14 & 18 & $5,{ }^{a} 13^{c}$ & Percutaneous & $29 \%(4 / 14)^{d}$ & Bleeding $(n=2), \operatorname{HTX}(n=2)$, AV shunt $(n=1)$ \\
\hline Lencioni et a $\left.\right|^{24}$ & 26 & 29 & $29^{a}$ & $\mathrm{~N} / \mathrm{A}$ & $8 \%(2 / 26)^{d}$ & HTX $(n=I)$, hepatic decompensation $(n=I)$ \\
\hline Dollinger et $\mathrm{al}^{16}$ & 56 & 114 & $45,{ }^{a} 62,{ }^{c} 7^{b}$ & Percutaneous & $26 \%(22 / 85)^{d}$ & $\begin{array}{l}\text { Abscess }(n=4) \text {, bleed }(n=7) \text {, renal failure }(n=1) \text {, } \\
\text { portal vein branch thrombosis }(n=5) \text {, PTX }(n=3) \text {, } \\
\text { arterial shunt }(n=3) \text {, neurologic deficit from } \\
\text { patient positioning }(n=2)\end{array}$ \\
\hline 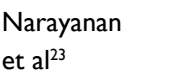 & 21 & 29 & $29^{a}$ & Percutaneous & $11 \%(3 / 28)^{d}$ & $\operatorname{HTX}(n=I), \operatorname{PTX}(n=I)$, effusion $(n=I)$ \\
\hline
\end{tabular}

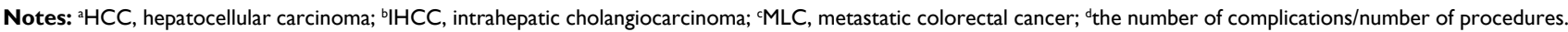
Abbreviations: HTX, hemothorax; IRE, irreversible electroporation; N/A, not available; PTX, pneumothorax; PVT, portal vein thrombosis; UTI, urinary tract infection.

pneumothoraces $(\mathrm{n}=2)$. Abscesses occurred more frequently in patients with bilioenteric anastomoses ( 3 of 4 patients).

By using a multi-institutional prospective registry, Cannon et $a{ }^{19}$ reported on the safety of IRE. They assessed the safety of 48 IRE procedures performed on 44 patients and reported a total of 9 adverse events occurring following $5(10 \%)$ procedures within 90 days. These included leukocytosis $(n=1)$, urinary tract infection $(n=1)$, dehydration $(n=1)$, biliary stent occlusion $(n=1)$, cholangitis $(n=1)$, renal failure $(\mathrm{n}=1)$, abdominal pain $(\mathrm{n}=1)$, flank pain $(\mathrm{n}=1)$, and neurogenic bladder $(n=1)$. The authors noted that they had no late occurrences of biliary stricture of portal vein thrombosis.

In a prospective, nonrandomized study, Cheung et al ${ }^{20}$ reported their experience in treating $18 \mathrm{HCC}$ lesions in 11 patients. No serious complications were reported despite 7 of 18 lesions being adjacent to important structures or organs (ie, portal vein, hepatic vein, heart, colon, duodenum, and gallbladder). They described treatment-related pain in $64 \%$ (7 of 11) of patients and transient urinary retention in $36 \%$ (4 of 11) of patients. In addition, the authors noted that all of the patients with urinary retention had a history of prostate hypertrophy.

In an article on safety in cirrhotic patients, Bhutiani et $\mathrm{al}^{21}$ treated 55 patients with $\mathrm{HCC}$ and Child-Pugh class B cirrhosis. They delivered treatments with either IRE $(n=30)$ or MWA $(\mathrm{n}=25)$. In comparing outcomes in these cirrhotic patients, they noted that the IRE was statistically superior with regard to length of stay ( 1 day versus 2 days; $p=0.05$ ) and 90 -day readmission ( $13 \%$ versus $36 \% ; p=0.03$ ). Complications occurred less frequently in IRE ( $27 \%$ versus $76 \%)$. They noted pleural effusions (17\%, 5 of 30$)$, a portal vein thrombosis (3\%, 1 of 30), and ascites (17\%, 5 of 30$)$. All of these complications were statistically less common than in the MWA group, suggesting IRE may have a better safety profile.

Eller et $\mathrm{a}^{22}$ described their initial experience in using IRE for malignant liver lesions. Of 18 lesions ablated in 14 patients, the authors reported a complication in $29 \%$ of patients ( 4 of 14). These included 1 patient with hemoperitoneum that resolved with conservative management, 1 patient with hemoperitoneum that required surgical intervention and an arterial-portal shunt requiring embolization, and 2 patients with right-sided hemothoraces.

In a comparison study on pain following treatment with $\operatorname{IRE}(\mathrm{n}=21)$ or RFA $(\mathrm{n}=21)$, Narayanan et $\mathrm{al}^{23}$ retrospectively evaluated postprocedure pain in patients with HCC. The authors found pain to be equivalent between the 2 treatment modalities. In the IRE group, they had a $10.7 \%$ complication rate in 28 treatments with IRE. These were comprised of hemothorax $(n=1)$, pneumothorax $(n=1)$, and a pleural effusion ( $\mathrm{n}=1)$.

In a multicenter prospective trial, Lencioni et $\mathrm{al}^{24}$ reported the outcomes of 21 patients undergoing 29 ablations for HCC. Although the manuscript has not yet been published, their abstract described a complication rate of $8 \%$ (2 of 26), which includes a hemothorax requiring drainage $(n=1)$ and transient hepatic decompensation $(\mathrm{n}=1)$. 
Particularly important to the treatment of HCC are the numerous studies showing that properly selected patients with cirrhosis can tolerate IRE. . $^{19,21,23,25-27}$ Although some studies limited analysis to Child-Pugh class A and B, ${ }^{21,26,27}$ many studies do not discuss specifics of patient selection in the setting of cirrhosis. Although the changes in electrical delivery have not been fully elucidated in the cirrhotic liver, Abdelsalam et $\mathrm{al}^{28}$ used a porcine model to compare IRE in cirrhotic livers to a control. The authors did not show any difference in size of ablation or resistance in the cirrhotic livers, and histopathologic response to IRE was similar between the groups.

The data presented earlier would suggest that IRE carries similar risks to other ablation techniques. After the implementation of cardiac synchronization, ${ }^{13}$ complications of IRE are primarily related to electrode placement. Using RFA in cirrhotic patients carries a morbidity of $28 \%,{ }^{3}$ and although IRE requires more electrodes for the delivery of a therapy, it carries a comparable morbidity profile ranging from $0 \%$ to $27.5 \%$ (Table 1). The data suggest that IRE does, in fact, appear to be safe adjacent to critical structures such as the bile duct with less apparent collateral damage compared to thermal ablation.

\section{Efficacy}

The ability of IRE to effectively ablate liver tissue has been shown in early preclinical work..$^{6-8,29-32}$ The earliest report of IRE for ablation of HCC in vivo was obtained from Guo et al. ${ }^{33}$ The authors created a hepatoma model in 30 SpragueDawley rats. Animals either underwent IRE with eight 100-ms pulses of $2500 \mathrm{~V} / \mathrm{cm}$ or served as controls. Complete pathologic response was found in 9 of 10 treated rats when sacrificed at $7-15$ days post-IRE.

Niessen et $\mathrm{al}^{25}$ reported their results in a prospective, single-center trial conducted to look at risk factors for local recurrence. They performed IRE on $22 \mathrm{HCC}$ lesions, and they described a local recurrence rate of $13.6 \%$ ( 3 of 22 ) within 6 months (Table 2). On a further analysis, they determined that HCC was less likely to have local recurrence than cholangiocarcinoma (33.3\%) and metastatic colorectal cancer (37.5\%). In addition, they found that larger tumor volumes $\left(>5 \mathrm{~cm}^{3}\right)$ portended early local recurrence.

Through a multi-institutional prospective registry, Cannon et $\mathrm{l}^{19}$ analyzed the tumor characteristics, complications, and local recurrence-free survival of 44 patients undergoing 48 IRE procedures over a 2-year period. All the patients had centrally located tumors in close proximity to major vascular

Table 2 Outcomes of hepatocellular carcinoma (HCC) ablation studies

\begin{tabular}{|c|c|c|c|c|c|c|c|c|c|c|}
\hline \multirow[t]{2}{*}{ Reference } & \multirow{2}{*}{$\begin{array}{l}\text { Number } \\
\text { of HCC } \\
\text { patients }\end{array}$} & \multirow{2}{*}{$\begin{array}{l}\text { Number } \\
\text { of HCC } \\
\text { lesions }\end{array}$} & \multirow{2}{*}{$\begin{array}{l}\text { Lesion } \\
\text { size }(\mathrm{cm}) \\
\begin{array}{l}\text { Median } \\
\text { (range) }\end{array}\end{array}$} & \multicolumn{4}{|c|}{ Treatment parameters } & \multicolumn{2}{|l|}{ Response } & \multirow{2}{*}{$\begin{array}{l}\begin{array}{l}\text { Local } \\
\text { recurrence }\end{array} \\
6 \text { months }\end{array}$} \\
\hline & & & & $\begin{array}{l}\text { Pulse } \\
\text { length } \\
(\mathrm{ms})^{\mathrm{d}}\end{array}$ & $\begin{array}{l}\text { Electricity } \\
\text { delivery }^{d}\end{array}$ & Pulses $^{d}$ & Approach & $\overline{\mathrm{CR}}$ & PR & \\
\hline $\begin{array}{l}\text { Cheng } \\
\text { et } \mathrm{al}^{27}\end{array}$ & 6 & 6 & $2.2(0.6-2.6)$ & $90-100$ & $1500 \mathrm{~V} / \mathrm{cm}$ & & Percutaneous & $\begin{array}{l}100 \%(6 / 6)^{\mathrm{a}} \\
83 \%(5 / 6)^{\mathrm{b}}\end{array}$ & $17 \%(1 / 6)^{b}$ & \\
\hline $\begin{array}{l}\text { Gonzalez- } \\
\text { Beicos } \\
\text { et } \mathrm{al}^{36}\end{array}$ & $\mathrm{~N} / \mathrm{A}$ & 4 & $N / A$ & $\mathrm{~N} / \mathrm{A}$ & $\mathrm{N} / \mathrm{A}$ & $\mathrm{N} / \mathrm{A}$ & Percutaneous & $\begin{array}{l}25 \%(I / 4) ;^{a} \\
25 \%(I / 4)^{b}\end{array}$ & $50 \%(2 / 4)^{\mathrm{b}}$ & \\
\hline $\begin{array}{l}\text { Niessen } \\
\text { et } \mathrm{a}^{25}\end{array}$ & $\mathrm{~N} / \mathrm{A}$ & 22 & $4.6^{3}$ & N/A & $1000-3000 \mathrm{~V}$ & $70-110$ & Percutaneous & & & $13.6 \%(3 / 22)^{\mathrm{a}}$ \\
\hline $\begin{array}{l}\text { Cannon } \\
\text { et al }{ }^{19}\end{array}$ & $\mathrm{~N} / \mathrm{A}$ & 14 & $2.1(1.3-4.5)$ & $20-100$ & $3000 \mathrm{~V}$ & 90 & NA & $100 \%(14 / 14)^{a}$ & & \\
\hline $\begin{array}{l}\text { Cheung } \\
\text { et } \mathrm{al}^{20}\end{array}$ & II & 18 & $2.4(I-6.1)$ & 70 & $1500-3000 \mathrm{~V}$ & 90 & Percutaneous & $72 \%(\mid 3 / / 8)^{\mathrm{a}, \mathrm{c}}$ & & \\
\hline $\begin{array}{l}\text { Bhutiani } \\
\text { et } \mathrm{al}^{21}\end{array}$ & 30 & $N / A$ & $3(2-3.3)$ & $20-100$ & $\mathrm{~N} / \mathrm{A}$ & 90 & Surgical & $97 \%(29 / 30)^{\mathrm{a}}$ & & \\
\hline Eller et $\mathrm{al}^{22}$ & 4 & 5 & $\begin{array}{l}\mathrm{N} / \mathrm{A} \\
(2.1-2.3)\end{array}$ & 100 & $1500 \mathrm{~V} / \mathrm{cm}$ & 90 & Percutaneous & $100 \%(5 / 5)^{\mathrm{a}}$ & & $25 \%(11 / 4)^{a}$ \\
\hline $\begin{array}{l}\text { Granata } \\
\text { et } \mathrm{a}^{34}\end{array}$ & 20 & 24 & $2(1-3)$ & $\mathrm{N} / \mathrm{A}$ & N/A & $\mathrm{N} / \mathrm{A}$ & Percutaneous & $92 \%(22 / 24)^{\mathrm{a}}$ & $8 \%(2 / 24)^{a}$ & \\
\hline $\begin{array}{l}\text { Sugimoto } \\
\text { et } \mathrm{a}^{35}\end{array}$ & 5 & 6 & I.8 (I.I-2.8) & 70 & $1500-1800 \mathrm{~V} / \mathrm{cm}$ & 90 & Percutaneous & $83 \%(5 / 6)^{a}$ & & $17 \%(1 / 6)^{a}$ \\
\hline $\begin{array}{l}\text { Lencioni } \\
\text { et } \mathrm{al}^{24}\end{array}$ & 26 & 29 & $\mathrm{~N} / \mathrm{A}$ & $\mathrm{N} / \mathrm{A}$ & $\mathrm{N} / \mathrm{A}$ & $N / A$ & $\mathrm{~N} / \mathrm{A}$ & $77 \%(20 / 26)^{a}$ & $15 \%(4 / 26)^{\mathrm{a}}$ & \\
\hline Padia et a ${ }^{26}$ & 20 & N/A & $2(1-3.3)$ & $20-100$ & $3000 \mathrm{~V}$ & 90 & Mixed & $90 \%(18 / 20)^{\mathrm{a}}$ & $10 \%(2 / 20)^{\mathrm{a}}$ & \\
\hline
\end{tabular}

Notes: Complete response (CR), partial response (PR) and a designation of aradiographic response and bathologic response at time of liver explant for liver transplantation. c93\% CR for tumors $<3 \mathrm{~cm}$. dData is presented as range or number, as some institutions use a range of pulses whilst some use one pulse. Abbreviation: N/A, not available. 
structures, biliary structures, or adjacent organs. The most common diagnosis was metastatic colorectal cancer $(n=20)$ followed in frequency by HCC $(n=14)$ and others $(n=10)$. Of the patients with HCC, 75\% were classified as Child-Pugh class $\mathrm{A}$, and the remaining as class $\mathrm{B}$. The majority $(76.5 \%)$ of IRE was performed percutaneously. Of the HCC lesions, local recurrence-free survival at 3, 6, and 12 months was $90 \%, 90 \%$, and $50 \%$, respectively.

Cheung et $\mathrm{al}^{20}$ reported their experience in treating 11 HCC patients with IRE. A total of 18 ablations were performed with an average tumor diameter of $2.44 \mathrm{~cm}$. During a mean follow-up of 18 months, 6 of 11 patients required repeat IRE for residual or recurrent disease. Outcomes were assessed with triple-phase CT. Ablation was considered successful if there was no enhancing tumor on triple-phase $\mathrm{CT}$, and the ablation zone included the entire tumor. Local disease control was achieved in 13 of 18 (72\%) lesions. Tumor size $>3 \mathrm{~cm}$ was the biggest risk for local recurrence in this series. One lesion required retreatment before complete response (CR) was observed. Of the 5 lesions incompletely ablated, 4 were $>3 \mathrm{~cm}$ in diameter, and the remaining lesion was $2.9 \mathrm{~cm}$. CR, defined as no residual or recurrent disease at a follow-up of at least 6 months, was successful with IRE of small HCC tumors $(\leq 3 \mathrm{~cm})$ in 13 of 14 lesions $(93 \%)$. Overall, the authors reported a local recurrence-free period of $18 \pm 4$ months and a distant recurrence-free period of $14 \pm 6$ months in the 6 patients who had a CR.

Bhutiani et $\mathrm{al}^{21}$ reported their experience in treating 55 patients with Child-Pugh class B HCC. They treated with either IRE $(n=30)$ or MWA $(n=25)$. The decision of MWA or IRE was made based on anatomic tumor location and adjacent structures such as major vessels or the biliary tract. Assessment of outcomes was based on blinded radiology reads on follow-up triple-phase CT at 1, 3, and 6 months. The authors noted that prior treatment to the liver had been attempted in most patients in both groups ( $84 \%$ MWA and $70 \%$ IRE). The majority of procedures for MWA and IRE were performed laparoscopically. Mean tumor size for MWA and IRE was not different (mean $3.2 \mathrm{~cm}$ versus $3.0 \mathrm{~cm}$, respectively). IRE was more likely to be used in proximity to the hepatic veins $(57 \%$ versus $16 \%)$ and in proximity to major portal inflow (63\% versus $0 \%$ ). Six-month success rates of $97 \%$ were noted in IRE compared to $100 \%$ in MWA as assessed by triple-phase CT. One local recurrence in the IRE group was in a $3.8-\mathrm{cm}$ lesion.

Describing their initial experience by using IRE for malignant liver lesions, Eller et $\mathrm{al}^{22}$ ablated $5 \mathrm{HCC}$ lesions in 4 patients. Lesion size ranged from $2.1 \mathrm{~cm}$ to $2.3 \mathrm{~cm}$. All
4 patients had initial success after IRE, and local recurrence occurred in 1 lesion at 14-month follow-up. Although the remaining 3 patients had no local recurrence, they all had new lesions in other locations noted during their follow-up period, which ranged from 4 to 19 months.

Granata et $\mathrm{al}^{34}$ reported the outcomes of $24 \mathrm{HCC}$ lesions in 20 parents treated with IRE. By 6-month follow-up MRI, contrast-enhanced ultrasound (CEUS) and CT showed 22 necrotic areas and 2 residual tumors in ablated areas ( $\mathrm{CR}=91.7 \%$, progressive disease [PD] 8.3\%). The authors did not show any statistically significant difference in the ability of MRI, CEUS, and CT to determine CR, partial response $(\mathrm{PR})$, and $\mathrm{PD}$.

In a small series from Japan, Sugimoto et $\mathrm{al}^{35}$ described their results of treating 5 patients with $6 \mathrm{HCC}$ lesions with IRE. Tumors ranged in size from $1.1 \mathrm{~cm}$ to $2.8 \mathrm{~cm}$. They reported 1 persistent lesion on MRI at Day 7, with a technical success rate of $83 \%$ ( 5 of 6 tumors). They attributed this persistent disease to a technical failure, as they did not deliver additional pulses due to an inability to visualize the tip of one of the probes in close proximity to the hepatic artery. They reported no major complications or deaths.

The most anticipated data on IRE and HCC are the final results from a prospective, multicenter study designed to evaluate safety and efficacy of IRE as a first-line treatment of biopsy-proven, early-stage HCC (clinicaltrials.gov ID: NCT01078415). Lencioni et $\mathrm{al}^{24}$ presented their abstract in 2012 showing that, of the 29 tumors in 26 patients, they had a 1 -month CR rate of $77 \%$ (20 of 26), PR rate of $15 \%$ (4 of 26), stable disease rate of $4 \%$ ( 1 of 26 ), and PD rate of $4 \%$ ( 1 of 26). The final results of this study have yet to be published.

In a study primarily assessing post-IRE MRI findings, Padia et $\mathrm{al}^{26}$ reported their success in using IRE in 20 patients with HCC. Although the primary focus was not on efficacy, they reported a 1-month CR rate of 90\% (18 of 20) and PR rate of $10 \%$ ( 2 of 20$)$.

The same group reported their experience in treating $6 \mathrm{HCC}$ patients with IRE as a bridging therapy prior to liver transplantation. Cheng et $\mathrm{al}^{27}$ described an ablate and resect study with a mean interval between the IRE and explant of 10 months; 4 of the 6 were Child-Pugh class A, and the remaining 2 were class $B$. None of the tumors were amenable to thermal ablation due to being adjacent to large $(>3 \mathrm{~mm})$ vessels, central bile ducts, or visceral organs (eg, stomach and colon). All treated tumors demonstrated a CR on followup imaging, defined as no tumor enhancement on imaging. On histologic evaluation, 5 of the 6 had no viable carcinoma cells. One patient with a $6-\mathrm{mm}$ lesion treated with a single 
bipolar probe, when 2 probers were not technically feasible, had $<5 \%$ carcinoma cells at the periphery of a $3.5-\mathrm{cm}$ area. The bipolar probe was intended for margin accentuation or extremely small tumors as it only has a 5-mm zone of ablation. This probe is no longer available from the manufacturer. This is one of the most promising studies to show that IRE does result in histologic tumor response in humans with HCC. One limitation of the study was that half of the tumors $(n=3)$ were previously treated (transarterial chemoembolization [TACE], $\mathrm{n}=2$; and 1 with chemoembolization and RFA). This limits the ability to attribute the histologic response in those patients solely to IRE. This study is also important in that it suggest that imaging may overestimate pathologic CR.

In an ablate and resect study where patients did not undergo prior therapies, Gonzalez-Beicos et $\mathrm{al}^{36}$ compared radiographic response and histologic response in a retrospective review. Their report included patients who underwent IRE followed by either surgery or transplantation. None of these patients had prior treatments for their lesions. Average imaging follow-up after IRE was 203 (range 55-429) days, and average time between IRE and surgery was 358 (range 78-527) days. Of the $4 \mathrm{HCC}$ lesions treated, follow-up imaging showed $\mathrm{CR}$ in 1 patient $(25 \%)$ and stable disease in $3(75 \%)$. Using hematoxylin and eosin (H\&E) staining, histologic evaluation found CR in $1(24 \%)$ lesion, PR in $2(50 \%)$, and no response in $1(25 \%)$. Despite all tumors being close to vascular structures, there was no evidence of post-IRE complications, and vessels remained patent and intact after IRE.

Unfortunately, larger retrospective studies ${ }^{13}$ and retrospective analysis of prospective data ${ }^{19}$ include combined outcome data of different types of tumors or even ablated organs. These data are difficult to interpret given the breadth of different diseases and/or indications, which are lumped together.

The studies that report HCC-specific outcomes do seem to show encouraging response rates with most $\mathrm{CR}$ rates ranging between $72 \%$ and $100 \%$ and 6-month local recurrence rate ranging from $13 \%$ to $25 \%$, however follow-up is limited to short intervals and confounded by additional tumor-directed therapies such as thermal ablation or TACE before or after the IRE procedure. Good outcomes were achieved in smaller tumors with most studies using IRE for lesions less than $3 \mathrm{~cm}$ and some studies even showed decreased response rates for tumors larger than this. Many of the encouraging outcomes described earlier were safely achieved in lesions with close proximity to either portal structures or large vessels, which have become the notable advantage for IRE. There remains limited data in the literature regarding the long-term effects of IRE alone on HCC.

\section{Challenges for IRE in the treatment of HCC \\ Determining effective ablation}

Histologic assessment of effective ablation following IRE needs to be done differently than following thermal ablation. With RFA, for example, histopathologic response can be seen immediately as a zone of coagulation necrosis. ${ }^{11}$ Although hemorrhagic necrosis and edema can be seen on H\&E staining after IRE immediately following treatment, ${ }^{8,30,32}$ this does not necessarily correlate with the zone of predictable cell death. ${ }^{30}$ It has been suggested that it may take up to 3 days for $H \& E$ staining to reliably predict the zone of ablation following IRE. ${ }^{37}$

Tissue destruction after IRE appears to occur in part via apoptosis. Evidence supporting this has shown intracellular pro-apoptotic oncoprotein (BCL-2) staining in IRE-ablated tissue that was not evident in nonablated tissues. ${ }^{32}$ This may account for the inability to consistently determine the zone of ablation on immediate H\&E staining. A more reliable method for determining nonviability may be the use of triphenyltetrazolium chloride (TTC) staining to determine the histologic area of anticipated cell death. TTC has been used to predict the zone of irreversible myocardial ischemia before histologic evidence can be noted on H\&E staining. ${ }^{38}$ TTC staining can show the zone of IRE in as little as $15 \mathrm{~min}$ after the treatment of porcine liver. ${ }^{6}$

Relying on imaging may also be unreliable. As was seen in RFA, ${ }^{2,3}$ the post-IRE imaging may overestimate pathologic $\mathrm{CR} .{ }^{27}$ Accordingly, further investigations are needed to establish the true CR rates following the delivery of IRE therapy.

Given the limitations of an immediately evident histologic response to IRE, patients treated with IRE as a bridge to liver transplantation can afford an opportunity to understand the true pathologic response rate following IRE for the treatment of HCC. Cheng et $\mathrm{al}^{27}$ used this model and showed a CR in 5 of 6 patients; however, half of these patients received prior treatments including TACE and thermal ablation.

\section{Imaging of patients undergoing IRE}

Imaging of patients undergoing IRE presents unique challenges to the radiologist, which are just beginning to be understood. Familiarity with the expected posttreatment imaging appearance on CT and MRI is critical for accurate 
assessment of procedural success, as well as for long-term surveillance of the ablation zone for recurrent disease. As with other percutaneous therapies, routine assessment using only size criteria (Response Evaluation Criteria in Solid Tumors) was insufficient for analysis. Multiphase, contrastenhanced imaging with careful adherence to best practice technique is essential.

Preprocedural imaging should consist of either multiphase contrast-enhanced CT or MRI. At the authors' institution (Rhode Island Hospital, Providence, RI, USA), MRI is preferred due to its increased sensitivity for additional sites of intrahepatic disease as well as the potential utility of diffusion-weighted imaging (DWI) to aid in later assessment of treatment efficacy. CT is typically reserved for patients with contraindication to MRI (cardiac devices) or difficulty with breath-holding.

Regardless of imaging modality, intravenous (IV) contrast, and multiphasic scanning is essential for accurate procedural planning. At a minimum, images should be acquired before IV administration of contrast, as well as in the late arterial and delayed phases. The late arterial phase, characterized by the enhancement of the portal venous system but not the hepatic veins, is the most sensitive for detection of HCC as tumors parasitize blood flow from the hepatic arteries, whereas the normal liver parenchyma receives the bulk of its blood supply from the portal vein. Delayed imaging adds to specificity by identifying contrast washout. According to guidelines published by the American Association for the Study of Liver Diseases, in patients with underlying liver disease, arterial phase enhancement and washout on delayed imaging are diagnostic of $\mathrm{HCC}{ }^{39}$

At the authors' institution (Rhode Island Hospital, Providence, RI, USA), imaging is performed 4 weeks following IRE. This examination essentially serves as a new baseline to which we can compare future examinations. Imaging of the liver is then repeated at 3-month intervals. The authors do not routinely image patients prior to 1 month as in vitro studies have shown that identifying residual or recurrent disease in the acute phase following IRE may be difficult or impossible. ${ }^{40}$ In addition, in up to $73 \%$ of cases, IRE results in gas accumulation within the ablation zone, which may be mistaken for a hepatic abscess. ${ }^{41}$

As stated previously, an imaging diagnosis of HCC can be confidently made when a mass, in a patient with liver disease, demonstrates arterial phase enhancement and delayed washout. The conspicuity of HCC on T2-weighted images depends on technique, but lesions are commonly mildly bright when compared with liver parenchyma. Restricted diffusion, bright signal on DWI and dark signal on corresponding apparent diffusion coefficient (ADC), is also commonly seen. ${ }^{42}$

In studies that have performed MRI immediately following ablation, authors have described a well-demarcated ablation zone with lack of internal enhancement and a peripheral rim of thick enhancement. This thick rim of peripheral enhancement gradually diminished over time and may not be visible at 1-month follow-up. The size of the ablation zone gradually decreased over time, whereas the central area of the ablation zone tended to demonstrate decreased signal on T2-weighted images and increased signal on T1-weighted images when compared to baseline. ${ }^{26}$

By 1 month, the ablation zone was typically well demarcated from normal liver parenchyma and slightly larger than the original tumor. It demonstrated decreased signal on T2-weighted images and mildly increased signal on T1-weighted images. The pathophysiologic basis of these signal changes was not well understood. No internal or nodular enhancement should be detected, but a smooth rim of peripheral enhancement may be variably present.

The role of diffusion-weighted images is not clear. While many have tried to use DWI to assess efficacy of ablation and to detect recurrence, results have been variable. Granata et al did not find a significant change in the ADC of treated liver lesions at 1 month when compared to baseline MRI. ${ }^{43}$ However, other studies, including a study using IRE to treat locally advanced pancreatic cancer, have shown an increase in ADC following treatment to be a reliable indicator of treatment success. ${ }^{44}$ Further studies are needed to elucidate the role of this potentially powerful imaging technique.

When CT is used in place of MRI for posttreatment monitoring, the appearance of the ablated tumors mimics that of the pre- and postcontrast-enhanced MRI sequences. Prior to administration of contrast, the ablation zone may be either hypoattenuating or heterogeneously hyperattenuating (likely due to the presence of debris/necrosis). Following administration of contrast, there are no internal enhancement and a variable degree of smooth, peripheral enhancement. ${ }^{34}$

Whether MRI or CT is used for follow-up imaging, the ablation zone must be carefully inspected for local recurrence. The earliest sign of local recurrence is usually a peripheral, nodular focus of arterial enhancement, which will often lose signal/attenuation on delayed phase images. When the ablation zone is bright on precontrast T1-weighted images, subtracted images (postcontrast image minus precontrast image) may be useful to improve the conspicuity of enhancement. These peripheral nodules of enhancement may also exhibit restricted diffusion, although additional studies 
are necessary to assess the utility of this technique. Finally, the remaining liver parenchyma should be carefully inspected for new tumors in this at-risk patient population.

\section{Limitations of tumor size}

Like thermal ablation, the best results with IRE are seen after treating tumors $<3 \mathrm{~cm} .{ }^{19-21,27,45}$ Although ablation zones $>6$ $\mathrm{cm}$ have been achieved in animal models, ${ }^{32,46}$ clinical trials suggest unreliable oncologic efficacy with tumors $>3 \mathrm{~cm}$. Currently the sole platform available to deliver IRE therapy is the NanoKnife system. This system uses monopolar electrodes used in parallel, which creates dumbbell-shaped ablation zones (Figure 2). As the electrodes approach each other, the zone of ablation takes on a more spherical configuration. When the electrodes are separate by $>3 \mathrm{~cm}$, gaps are created in the areas or IRE. In addition, for tumors $>3 \mathrm{~cm}$, the probe configuration requires at least 4 electrodes. These electrodes need to be placed in parallel, and their positional configurations can quickly become complicated. ${ }^{19,21}$ This leaves little room for error and likely influences the inability to irreversibly electroporate larger tumors.

\section{Technically demanding procedure}

Further demands of IRE included the need for general anesthesia and paralysis, which can extend time needed to complete a procedure. $^{22}$ Several authors have noted the increased technical precision required to ablate tumors using IRE compared to RFA. ${ }^{22,35}$ RFA requires the placement of one large catheter within the center of the lesion to be ablated, whereas IRE requires the placement of multiple (2-5) probes, all in parallel and bracketed around the tumor to incorporate the lesion in the electrical field. This can be especially difficult with

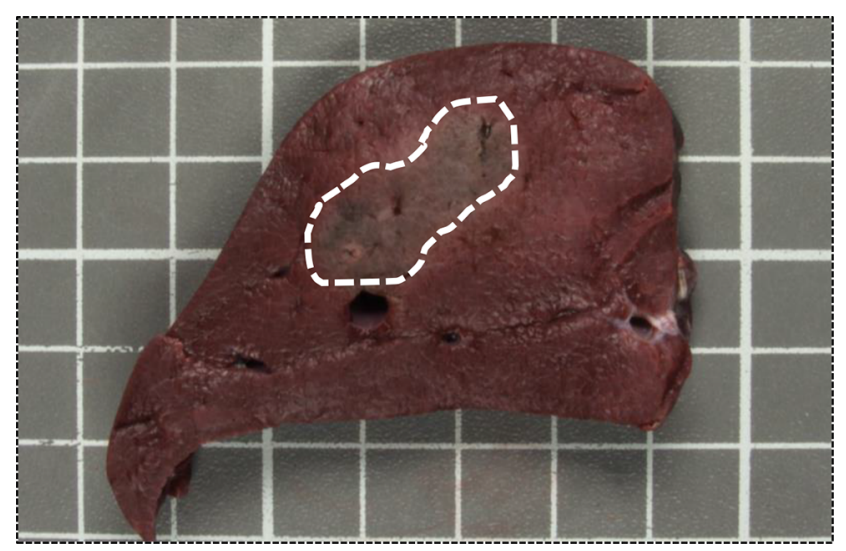

Figure 2 Swine liver stained with triphenyltetrazolium chloride.

Notes: Swine liver stained with triphenyltetrazolium chloride following irreversible electroporation with 2 monopolar electrodes. The dashed line highlights the dumbbell shape of the ablation zone. percutaneous probe placement in parallel around anatomic obstacles such as ribs. ${ }^{22}$ What adds to the unforgiving nature of IRE is that variance in probe placement is associated with incomplete ablation, ${ }^{7}$ which is only compounded by the flexible nature of the IRE probes. Due to these difficulties, some authors used the aid of a robot positioner (Perfint Maxio) and CT scan to confirm placement. ${ }^{45}$ Some of the technically demanding and hindering aspects of IRE could be remedied with a bipolar electrode. To the authors' knowledge, there are currently no commercially available bipolar IRE electrodes.

\section{Lack of investigational device exemptions (IDEs)}

To date, the authors are only aware of a single IDE for IRE. This was issued by the US Food and Drug Administration to study the use of NanoKnife for the treatment of prostate cancer. The inability to obtain an IDE for tissue-specific investigations into the NanoKnife has limited the ability of several centers within the USA to perform well-designed, prospective trials of the technology.

\section{Discussion}

Early clinical data suggested that in the hands of a skilled user, IRE may be equivalent to thermal ablation for the treatment of small $(<3 \mathrm{~cm}) \mathrm{HCCs}$ in appropriately selected cases. Given the paucity of long-term data demonstrating oncologic efficacy, the authors urge caution in the widespread application of IRE for the treatment of hepatomas that are amenable to alternative standard of care therapies such as liver transplantation, surgical resection, or thermal ablation. Currently, IRE fills a void and serves as a niche technology for ablating of small $(<3 \mathrm{~cm})$, unresectable tumors, not amenable to thermal ablation due to the abutment of major veins or hilar structures.

Despite these deficits in the literature, the advancement of IRE appears to be following the progression outlined in the Idea, Development, Exploration, Assessment, Long-term study recommendations. ${ }^{47}$ At the writing of the authors' last review, ${ }^{48}$ IRE remained within the Developmental (2a) stage of development; however, as seen in the data presented earlier, IRE has transitioned into the Exploration (2b) phase of development. In this phase of development, clinical outcomes now need to be a focus in order to assess the value in pursuing the technology. Through several studies, IRE has been shown to be safe, especially with regard to use within close proximity to the biliary and venous systems of the liver.

Standardized terminology and reporting criteria for IRE have been suggested, ${ }^{49}$ and future studies should incorporate this moving forward. Our focus on reporting efficacy should 
now transition from procedure-based reporting to diseasespecific reporting. In addition, longer-term outcomes should be emphasized so as to give a better idea of the benefits on overall outcomes. Looking into the future and given the niche role of IRE, collaborative prospective studies comparing IRE with an alternative treatment arm should be started early as patient accrual may be difficult. This would ideally be conducted in patients with $\mathrm{HCC}$, as outcomes appear to be more promising in this patient population. ${ }^{25}$

\section{Disclosure}

The authors report no conflicts of interest in this work.

\section{References}

1. Stewart BW, Wild CP, editors. World Cancer Report 2014. Available from: http://publications.iarc.fr/Non-Series-Publications/World-CancerReports/World-Cancer-Report-2014. Accessed October 1, 2016.

2. Pompili M, Mirante VG, Rondinara G, et al. Percutaneous ablation procedures in cirrhotic patients with hepatocellular carcinoma submitted to liver transplantation: assessment of efficacy at explant analysis and of safety for tumor recurrence. Liver Transpl. 2005;11(9):1117-1126.

3. Mazzaferro V, Battiston C, Perrone S, et al. Radiofrequency ablation of small hepatocellular carcinoma in cirrhotic patients awaiting liver transplantation: a prospective study. Ann Surg. 2004;240(5):900-909.

4. Diehn FE, Neeman Z, Hvizda JL, Wood BJ. Remote thermometry to avoid complications in radiofrequency ablation. J Vasc Interv Radiol. 2003;14(12):1569-1576

5. Carey RI, Leveillee RJ. First prize: direct real-time temperature monitoring for laparoscopic and CT-guided radiofrequency ablation of renal tumors between 3 and $5 \mathrm{~cm}$. J Endourol. 2007;21(8):807-813.

6. Charpentier KP, Wolf F, Noble L, Winn B, Resnick M, Dupuy DE. Irreversible electroporation of the liver and liver hilum in swine. $H P B$ (Oxford). 2011;13(3):168-173.

7. Charpentier KP, Wolf F, Noble L, Winn B, Resnick M, Dupuy DE. Irreversible electroporation of the pancreas in swine: a pilot study. $H P B$ (Oxford). 2010;12(5):348-351.

8. Lee EW, Wong D, Prikhodko SV, et al. Electron microscopic demonstration and evaluation of irreversible electroporation-induced nanopores on hepatocyte membranes. J Vasc Interv Radiol. 2012;23(1):107-113.

9. Golberg A, Bruinsma BG, Jaramillo M, Yarmush ML, Uygun BE. Rat liver regeneration following ablation with irreversible electroporation. Peer J. 2016;4:e1571.

10. Goldberg SN, Dupuy DE. Image-guided radiofrequency tumor ablation: challenges and opportunities — part I. J Vasc Interv Radiol. 2001; 12(9):1021-1032.

11. Goldberg SN, Hahn PF, Tanabe KK, et al. Percutaneous radiofrequency tissue ablation: does perfusion-mediated tissue cooling limit coagulation necrosis? J Vasc Interv Radiol. 1998;9(1):101-111.

12. Nielsen K, Scheffer HJ, Vieveen JM, et al. Anaesthetic management during open and percutaneous irreversible electroporation. Br JAnaesth. 2014;113(6):985-992.

13. Thomson KR, Cheung W, Ellis SJ, et al. Investigation of the safety of irreversible electroporation in humans. J Vasc Interv Radiol. 2011; 22(5):611-621.

14. Dollinger M, Müller-Wille R, Zeman F, et al. Irreversible electroporation of malignant hepatic tumors - alterations in venous structures at subacute follow-up and evolution at mid-term follow-up. PLoS One. 2015;10(8):e0135773.

15. Dollinger M, Zeman F, Niessen C, et al. Bile duct injury after irreversible electroporation of hepatic malignancies: evaluation of MR imaging findings and laboratory values. JVasc Interv Radiol. 2016;27(1):96-103.
16. Dollinger M, Beyer LP, Haimerl M, et al. Adverse effects of irreversible electroporation of malignant liver tumors under CT fluoroscopic guidance: a single-center experience. Diagn Interv Radiol. 2015;21(6):471-475.

17. Scheffer HJ, Nielsen K, De Jong MC, et al. Irreversible electroporation for nonthermal tumor ablation in the clinical setting: a systematic review of safety and efficacy. J Vasc Interv Radiol. 2014;25(7):997-1011.

18. Niessen C, Beyer LP, Pregler B, et al. Percutaneous ablation of hepatic tumors using irreversible electroporation: a prospective safety and midterm efficacy study in 34 patients. JVasc Interv Radiol. 2016;27(4): 480-486.

19. Cannon R, Ellis S, Hayes D, Narayanan G, Martin RCG. Safety and early efficacy of irreversible electroporation for hepatic tumors in proximity to vital structures. J Surg Oncol. 2013;107(5):544-549.

20. Cheung W, Kavnoudias H, Roberts S, Szkandera B, Kemp W, Thomson KR. Irreversible electroporation for unresectable hepatocellular carcinoma: initial experience and review of safety and outcomes. Technol Cancer Res Treat. 2013;12(3):233-241.

21. Bhutiani N, Philips P, Scoggins CR, McMasters KM, Potts MH, Martin RCG. Evaluation of tolerability and efficacy of irreversible electroporation (IRE) in treatment of Child-Pugh B (7/8) hepatocellular carcinoma (HCC). HPB (Oxford). 2016;18(7):593-599.

22. Eller A, Schmid A, Schmidt J, et al. Local control of perivascular malignant liver lesions using percutaneous irreversible electroporation: initial experiences. Cardiovasc Intervent Radiol. 2014;38(1): $152-159$.

23. Narayanan G, Froud T, Lo K, Barbery KJ, Perez-Rojas E, Yrizarry J. Pain analysis in patients with hepatocellular carcinoma: irreversible electroporation versus radiofrequency ablation - initial observations. Cardiovasc Intervent Radiol. 2013;36(1):176-182.

24. Lencioni R, Izzo F, Crocetti L, et al. A prospective, multicenter phase II clinical trial using irreversible electroporation for the treatment of early stage HCC. Scientific Session 18 Interventional Oncology: Chemoembolization III. J Vasc Interv Radiol. 2012;23:1114.

25. Niessen C, Igl J, Pregler B, et al. Factors associated with short-term local recurrence of liver cancer after percutaneous ablation using irreversible electroporation: a prospective single-center study. JVasc Interv Radiol. 2015;26(5):694-702.

26. Padia SA, Johnson GE, Yeung RS, Park JO, Hippe DS, Kogut MJ. Irreversible electroporation in patients with hepatocellular carcinoma: immediate versus delayed findings at MR imaging. Radiology. 2016;278(1):285-294.

27. Cheng RG, Bhattacharya R, Yeh MM, Padia SA. Irreversible electroporation can effectively ablate hepatocellular carcinoma to complete pathologic necrosis. J Vasc Interv Radiol. 2015;26(8):1184-1188.

28. Abdelsalam ME, Chetta JA, Harmoush S, et al. Irreversible electroporation (IRE) in cirrhotic liver: preliminary experience in a large animal model. J Vasc Interv Radiol. 2013;24(4):S46.

29. Al-Sakere B, André F, Bernat C, et al. Tumor ablation with irreversible electroporation. PLoS One. 2007;2(11):e1135.

30. Rubinsky B, Onik G, Mikus P. Irreversible electroporation: a new ablation modality-clinical implications. Technol Cancer Res Treat. 2007;6(1):37-48.

31. Bertacchini C, Margotti PM, BergaminiE, LodiA, Ronchetti M, Cadossi R. Design of an irreversible electroporation system for clinical use. Technol Cancer Res Treat. 2007;6(4):313-320.

32. Lee EW, Loh CT, Kee ST. Imaging guided percutaneous irreversible electroporation: ultrasound and immunohistological correlation. Technol Cancer Res Treat. 2007;6(4):287-294.

33. Guo Y, Zhang Y, Klein R, et al. Irreversible electroporation therapy in the liver: longitudinal efficacy studies in a rat model of hepatocellular carcinoma. Cancer Res. 2010;70(4):1555-1563.

34. Granata V, de Lutio di Castelguidone E, Fusco R, et al. Irreversible electroporation of hepatocellular carcinoma: preliminary report on the diagnostic accuracy of magnetic resonance, computer tomography, and contrast-enhanced ultrasound in evaluation of the ablated area. Radiol Med. 2016;121(2):122-131. 
35. Sugimoto K, Moriyasu F, KobayashiY, et al. Irreversible electroporation for nonthermal tumor ablation in patients with hepatocellular carcinoma: initial clinical experience in Japan. Jpn J Radiol. 2015;33(7):424-432.

36. Gonzalez-Beicos A, Venkat S, Songrug T, et al. Irreversible electroporation of hepatic and pancreatic malignancies: radiologic-pathologic correlation. Tech Vasc Interv Radiol. 2015;18(3):176-182.

37. Chunlan J, Davalos RV, Bischof JC. A review of basic to clinical studies of irreversible electroporation therapy. IEEE Trans Biomed Eng. 2015;62(1):4-20.

38. Fishbein MC, Meerbaum S, Rit J, et al. Early phase acute myocardial infarct size quantification: validation of the triphenyl tetrazolium chloride tissue enzyme staining technique. Am Heart J. 1981;101(5):593-600.

39. Bruix J, Sherman M; American Association for the Study of Liver Diseases. Management of hepatocellular carcinoma: an update. Hepatology. 2011;53(3):1020-1022.

40. Arena CB, Szot DCS, Garcia DPA, Rylander MN, Davalos RV. A three-dimensional in vitro tumor platform for modeling therapeutic irreversible electroporation. Biophys J. 2012;103(9):2033-2042.

41. Dollinger M, Jung EM, Beyer L, et al. Irreversible electroporation ablation of malignant hepatic tumors: subacute and follow-up CT appearance of ablation zones. J Vasc Interv Radiol. 2014;25(10):1589-1594.

42. Kele PG, van der Jagt EJ. Diffusion weighted imaging in the liver. World J Gastroenterol. 2010;16(13):1567-1576.
43. Granata V, Fusco R, Catalano O, et al. Percutaneous ablation therapy of hepatocellular carcinoma with irreversible electroporation: MRI findings. Am J Roentgenol. 2015;204(5):1000-1007.

44. Vroomen LGPH, Scheffer HJ, Melenhorst MCAM. MR and CT imaging characteristics and ablation zone volumetry of locally advanced pancreatic cancer treated with irreversible electroporation American Joint Committee on Cancer American Society of Anaesthesiologists. Eur Radiol. Epub 2016 Sep 22.

45. Thomson KR, Kavnoudias H, Neal RE. Introduction to irreversible electroporation-principles and techniques. Tech Vasc Interv Radiol. 2015;18(3):128-134.

46. Appelbaum L, Ben-David E, Faroja M, Nissenbaum Y, Sosna J, Goldberg $\mathrm{SN}$. Irreversible electroporation ablation: creation of large-volume ablation zones in in vivo porcine liver with four-electrode arrays. Radiology. 2014;270(2):416-424.

47. McCulloch P, Altman DG, Campbell WB, et al. No surgical innovation without evaluation: the IDEAL recommendations. Lancet. 2009; 374(9695):1105-1112.

48. Charpentier KP. Irreversible electroporation for the ablation of liver tumors: are we there yet? Arch Surg. 2012;147:1053-1061.

49. Wendler JJ, Fischbach K, Ricke J, et al. Irreversible electroporation (IRE): standardization of terminology and reporting criteria for analysis and comparison. Pol J Radiol. 2016;81:54-64.
Journal of Hepatocellular Carcinoma

\section{Publish your work in this journal}

The Journal of Hepatocellular Carcinoma is an international, peerreviewed, open access journal that offers a platform for the dissemination and study of clinical, translational and basic research findings in this rapidly developing field. Development in areas including, but not limited to, epidemiology, vaccination, hepatitis therapy, pathology and
Dovepress

molecular tumor classification and prognostication are all considered for publication. The manuscript management system is completely online and includes a very quick and fair peer-review system, which is all easy to use. Visit http://www.dovepress.com/testimonials.php to read real quotes from published authors. 